\title{
Available Simplified Semi-empirical Correlation to Predict Hollow Spray Cone Angle for Practical Pressure Swirl Atomizers with Viscous liquid
}

\author{
Lei Sun*1, Deng Tian², Yong Huang ${ }^{3}$, Shaolin Wang ${ }^{4}$, Zhilin Liü ${ }^{5}$ Bin Chen ${ }^{6}$, Wei Xiao7, \\ Xiang Feng ${ }^{8}$ \\ ${ }^{1}$ Sino-European Institute of Aviation Engineering (SIAE), Civil Aviation University of China, \\ Tianjin 300300, P.R. China \\ ${ }^{2}$ Sino-European Institute of Aviation Engineering (SIAE), Civil Aviation University of China, \\ Tianjin 300300, P.R. China \\ ${ }^{3}$ School of Energy and Power Engineering, Beihang University, Beijing 100191, P.R. China \\ ${ }^{4}$ Institute of Engineering Thermophysics, Chinese Academy of Sciences, Beijing 100190, \\ China \\ ${ }^{5}$ AVIC Chengdu Aircraft Industrial (Group) Co., Ltd, Chengdu 610092, Sichuan, P.R. China \\ ${ }^{6}$ China Construction Third Engineering Bureau Co., Ltd, Wuhan 430064, Hubei, P.R.China \\ ${ }^{7}$ AECC Hunan Aviation Powerplant Research Institute, Zhuzhou 421000, China \\ ${ }^{8}$ Patent Examination Cooperation (Tianjin) Center of the Patent Office, Tianjin 300304, P.R. \\ China \\ ${ }^{*}$ Corresponding author email: sunlei1988@buaa.edu.cn
}

\begin{abstract}
Pressure swirl atomizers are widely utilized in engineering, agriculture and medical treatment. The spray cone angle is one of the most important parameters for pressure swirl atomizers. The spatial distribution of sprays and droplets is closely dependent on the spray cone angle. In our previous study, an improved semi-empirical correlation is derived based on the rotational kinetic energy loss caused by the friction to predict the viscous spray cone angles. However, this correlation is relatively complicated and not convenient for the quick prediction of the hollow spray cone angle. In order to achieve quick prediction of the hollow spray cone angle with acceptable prediction accuracy, a simplified semi-empirical correlation is proposed in the present study. This simplified semi-empirical correlation is obtained by simplifying our previous correlation based on proper assumptions. Further, this simplified semi-empirical correlation could accommodate the effects of geometrical parameters, operating conditions and liquid properties on the spray cone angle. Besides, a series of experiments with variations of geometrical parameters, operating conditions and liquid properties have been done to validate the simplified semi-empirical correlation. The hollow spray cone angles predicted by this simplified semi-empirical correlation agree well with the experimental results. The prediction uncertainties could be within $\pm 15 \%$ for all cases.
\end{abstract}

Keywords: Semi-empirical correlation; Pressure swirl atomizer; Hollow spray cone angle; Viscous liquid.

\section{Introduction}

Pressure swirl atomizers are widely utilized in many fields, including combustion, process industries, agriculture and medical treatment, etc., due to simplified geometric configuration and good atomization performance [1, 2]. For example, pressure swirl atomizers are very common in medical treatment and attract more and more attentions, especially in 2020. During the COVID-19 pandemic, spraying the disinfectant liquid is the most important method to keep the environmental safety, especially in hospitals [3]. Besides, the aerosol 
inhalation treatment which mainly depends on atomizers is widely utilized for respiratory disease [4-6].

The spray cone angle is one of the most important parameters for pressure swirl atomizers. The spatial distribution of sprays and droplets is closely dependent on the spray cone angle. The hollow spray cone is the most typical spray formed by pressure swirl atomizer. For a typical pressure swirl atomizer, it is composed of a swirl chamber, a convergent duct and a discharge orifice [7]. The liquid is injected into the swirl chamber from the tangential holes. Then the liquid flows in the swirl chamber with both tangential and axial velocities. In the discharge orifice, the liquid flow accelerates due to the decreasing of the flow area. During this process, an air core is formed due to the effect of negative pressure in the region along the centreline of the pressure atomizer. Once the liquid flows into the external environment from the pressure swirl atomizer, a conical liquid sheet is formed [8]. The spray cone angle could be calculated based on the tangential and axial velocities at the plane of the outlet. In order to predict the spray cone angle, the liquid viscosity must be considered. At present, there are a series of correlations to predict the spray cone angle of the pressure swirl atomizer with viscous liquids. These available correlations are mainly obtained by fitting experimental data. Some improved correlations further try to bring the analyses of physical model. However, there are no available analytical results due to the complexity of internal flow inside the atomizer.

Giffen and Massey [9] carried out an experiment with the liquid kinematic viscosity changing from 2 to $50 \mathrm{cSt}$ and then obtained an empirical correlation between the spray cone angle and liquid kinematic viscosity. Rizk and Lefebvre [10] proposed an empirical correlation which could accommodate not only the effect of liquid viscosity but also the effects of liquid density, discharge orifice diameter, nozzle constant and injection pressure differential across nozzle. Zhang et al. [11] proposed an empirical correlation relating the spray cone angle with Reynolds number by Buckingham pi theorem [12] and experimental data. Rivas et al. [13] also claimed that they obtained a semi-empirical correlation to predict the spray cone angle with the consideration of liquid viscosity. In our previous study, an improved semi-empirical correlation is derived based on boundary layer theory to predict the viscous spray cone angles [8]. This improved semi-empirical correlation can reveal the effects of atomizer geometries, liquid properties and operating conditions. The prediction uncertainties of our improved semi-empirical correlation are within $\pm 10 \%$ by validating with experimental data. However, our original semi-empirical correlation is complicated and not convenient for the quick prediction. In practical application, the quick prediction of spray cone angle is important for design and optimization of a pressure swirl atomizer.

In this paper, an available simplified semi-empirical correlation is derived to predict the viscous hollow spray cone angle of pressure swirl atomizers based on proper assumptions and simplification of our original semi-empirical correlation. More new experimental data will be utilized to validate this available simplified semi-empirical correlation. Moreover, available prediction correlations will be introduced to make comparison with the available simplified semi-empirical correlation.

\section{Available simplified semi-empirical correlation}

Figure 1 shows the schematic of the geometric configuration of a typical pressure swirl atomizer in the present study. This pressure swirl atomizer is composed of a swirl chamber, a convergent duct and a discharge orifice. The tangential inlet ports are located at the bottom of the swirl chamber. The liquid from the liquid supply system upstream is injected into the swirl chamber through these tangential inlet ports. The radius and number of the tangential inlet ports are $r_{i}$ and $n$, respectively. The radius and length of the swirl chamber are $r_{s}$ and $L_{s}$; the angle and length of the convergent duct are $2 \alpha$ and $L_{c}$; the radius and length of the discharge orifice are $r_{o}$ and $L_{o}$, respectively. 

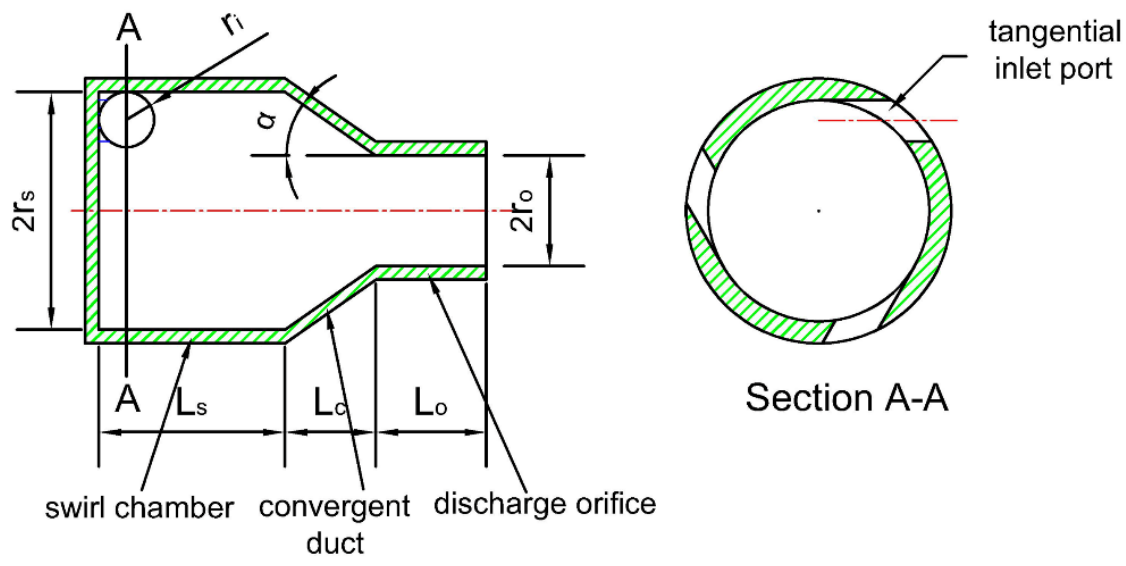

Section A-A

Figure 1. Schematic of the geometric configuration of the typical pressure swirl atomizer

Based on our previous study [8], an improved semi-empirical correlation that can be utilized to predict the viscous hollow spray cone angles is derived based on the rotational kinetic energy loss. The rotational kinetic energy loss caused by the friction can be calculated based on the boundary layer distributions. The expression of our original improved semiempirical correlation is

$$
2 \theta=\arctan \sqrt{\frac{1+\left(\frac{E_{A}-\Delta E_{s}-\Delta E_{c}}{E_{A}}\right)^{2}\left(\left.\frac{W_{\delta_{\theta}}}{U_{\delta_{x}}}\right|_{\text {invscid }}\right)^{2}}{\exp \left\{0.0027 v^{0.25} \frac{\rho^{0.25}}{\dot{m}^{0.25}} \frac{r_{o}^{2}\left(\frac{7}{8} r_{o}-\frac{7}{15} r_{a}\right)^{0.25}\left(n \pi r_{i}^{2}\right)^{0.25}}{r_{a} r_{s}^{0.5}\left(\frac{7}{9} r_{o}^{2}-\frac{7}{8} r_{a} r_{o}+\frac{7}{23} r_{a}^{2}\right)} \cdot L_{o}\right\}}}-1
$$

where

$$
\left\{\begin{array}{l}
E_{A}=\frac{\dot{m}^{2} R_{a}}{\rho A_{p}}=\frac{\dot{m}^{2} R_{a}}{n \pi \rho r_{i}^{2}} \\
\Delta E_{s}=0.0544 \frac{\dot{m}^{1.8} r_{s}^{0.4}}{n \pi \rho^{0.8} r_{i}^{2}}\left(1+\frac{9.8696 r_{s}^{4}}{\left(n \pi r_{i}^{2}\right)^{2}}\right)^{\frac{3}{8}} v^{\frac{1}{5}} L_{s}^{\frac{4}{5}} \\
\Delta E_{c}=0.855 \frac{\dot{m}^{1.75} r_{s}}{n \pi \rho^{0.75} r_{i}^{2}}\left(1+\frac{9.8696 r_{s}^{4}}{\left(n \pi r_{i}^{2}\right)^{2}}\right)^{\frac{3}{8}} v^{\frac{1}{4}} \frac{\left(0.465 \dot{m}^{-0.2} \rho^{0.2} r_{s}^{0.4} v^{0.2} L_{s}^{0.8}+r_{o}-r_{a}\right)^{\frac{1}{4}} \tan \alpha}{(\pi / 2)(1-X)^{1.5}} \\
\left.\frac{W_{\delta_{\theta}}}{U_{\delta_{x}}}\right|_{\text {invscid }}=\frac{\sqrt{r_{s}-r_{o}}}{\sqrt{K^{2}(1+\sqrt{X})^{2}(1+X)-\left(\pi^{2} / 4\right)(1-X)^{3}}} \\
K=\frac{A_{p}}{4 r_{s} r_{o}}=\frac{n \pi r_{i}^{2}}{4 r_{s} r_{o}} \\
X=\frac{r_{a}^{2}}{r_{o}^{2}} \\
K=\frac{(1-X)^{1.5}}{\sqrt{2} X} \\
r_{a}=r_{o}-1.13 r_{o}\left(\frac{m \mu}{\rho \Delta P r_{o}^{3}}\right)^{0.25}\left(\frac{L_{o}}{r_{o}}\right)^{0.6}
\end{array}\right.
$$


This improved semi-empirical correlation could systematically and quantitatively reveal the effects of the geometrical parameters, liquid properties and operating conditions on the spray cone angle. However, this semi-empirical correlation is rather complicated and not convenient for the quick prediction. Thus, an available simplified semi-empirical correlation is obtained based on the proper assumptions and simplifications.

Two proper assumptions are introduced as follows:

(I) For the practical pressure swirl atomizers, the angles of convergent duct are mainly around $90 \mathrm{deg}$. The aim of this assumption is to linearly account the rotational kinetic energy loss in the convergent duct into the rotational kinetic energy loss in the swirl chamber. This treatment could simplify the expression of the numerator in the term in the square root in Eq. (1).

(II) It should be noted that the present study is aimed at the hollow cone spray. For the practical pressure swirl atomizer, the thickness of the liquid sheet in the discharge orifice is of the order of magnitude of the discharge orifice diameter. This treatment could simplify the expression of the denominator in the term in the square root in Eq. (1). In fact, based on our experiment, the thickness of the liquid sheet in the discharge orifice is close to half of the discharge orifice diameter for the atomizers in the present study.

Based on assumption (I), it could be obtained

$$
\frac{E_{A}-\Delta E_{s}-\Delta E_{c}}{E_{A}}=1-\frac{\Delta E_{s}+\Delta E_{c}}{E_{A}}=1-\frac{\Delta E_{s}}{E_{A}}\left(1+M \frac{r_{s}-r_{o}}{L_{s} \tan \alpha}\right)
$$

where $M$ is a scaling factor determined by experimental results.

Based on assumption (II), it could be obtained

$$
0.0027 v^{0.25} \frac{\rho^{0.25}}{\dot{m}^{0.25}} \frac{r_{o}^{2}\left(\frac{7}{8} r_{o}-\frac{7}{15} r_{a}\right)^{0.25}\left(n \pi r_{i}^{2}\right)^{0.25}}{r_{a} r_{s}^{0.5}\left(\frac{7}{9} r_{o}^{2}-\frac{7}{8} r_{a} r_{o}+\frac{7}{23} r_{a}^{2}\right)} \cdot L_{o}=N\left(\mu \sqrt{\frac{\rho}{\Delta p}}\right)^{0.25} r_{o}^{-0.75} \cdot L_{o}
$$

where $N$ is a scaling factor determined by experimental results.

Then, Eq. (1) could be simplified as

$$
2 \theta=\arctan \sqrt{\frac{1+\left(1-10.7348 Q \frac{\rho^{0.1} \mu^{0.2} L_{s}^{0.8}}{\Delta p^{0.1}}\left(1+M \frac{r_{s}-r_{o}}{L_{s} \tan \alpha}\right)\right)^{2}\left(\left.\frac{W_{\delta_{\theta}}}{U_{\delta_{x}}}\right|_{\text {invscid }}\right)^{2}}{\exp \left\{N\left(\mu \sqrt{\frac{\rho}{\Delta p}}\right)^{0.25} r_{o}^{-0.75} \cdot L_{o}\right\}}}-1
$$

where $Q$ is a scaling factor determined by experimental results.

Compared with the expression of Eq. (1), the expression of Eq. (4) is simplified significantly. Further, Eq. (4) could be utilized to quick predict the spray cone angle when the geometrical parameters, liquid properties and operating conditions are fixed.

\section{Experiment setup}

The schematic of the experimental setup is shown in Figure 2. The schematics of the pressure swirl atomizers used in the present experiment are shown in Fig.1. The pressure swirl atomizers are made of plexiglass that can be utilized to achieve the visualization of the internal flow [14]. Five type pressure swirl atomizers with different length of swirl chamber are utilized. The detailed geometrical parameters of the pressure swirl atomizers are listed in Table 1. 


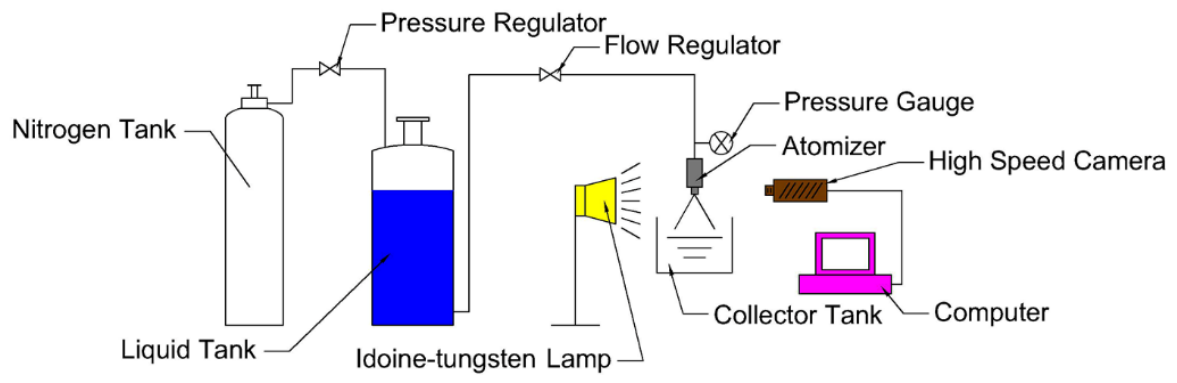

Figure 2. Schematic of experimental setup

Table 1 Detailed geometrical parameters of the pressure swirl atomizers

\begin{tabular}{|c|c|c|c|c|c|}
\hline Atomizer & 1 & 2 & 3 & 4 & 5 \\
\hline $\begin{array}{l}\text { Radius of tangential inlet ports, } r_{i} / \\
(\mathrm{mm})\end{array}$ & 0.5 & 0.5 & 0.5 & 0.5 & 0.5 \\
\hline Number of tangential inlet ports, $n$ & 3 & 3 & 3 & 3 & 3 \\
\hline Radius of swirl chamber, $r_{s} /(\mathrm{mm})$ & 2 & 2 & 2 & 2 & 2 \\
\hline Length of swirl chamber, $L_{s} /(\mathrm{mm})$ & 2.6 & 5 & 10 & 15 & 20 \\
\hline $2 \alpha /\left(^{\circ}\right)$ & 90 & 90 & 90 & 90 & 90 \\
\hline$L_{c} /(\mathrm{mm})$ & 1.5 & 1.5 & 1.5 & 1.5 & 1.5 \\
\hline$r_{o} /(\mathrm{mm})$ & 0.5 & 0.5 & 0.5 & 0.5 & 0.5 \\
\hline , $L_{o} /(\mathrm{mm})$ & 3 & 3 & 3 & 3 & 3 \\
\hline $\begin{array}{l}\text { Length } \\
\text { pressu } \\
\text { inlet p }\end{array}$ & 1.5 & 1.5 & 1.5 & 1.5 & 1.5 \\
\hline
\end{tabular}

The liquids utilized in these experiments are the mixtures of purified water and glycerol with four different volume ratios. The liquid viscosities of the mixtures at these four volume ratios are shown in Table 2.

Table 2 Liquid viscosities of the mixtures at these four volume ratios

\begin{tabular}{c|ccc}
\hline Mixture & $\begin{array}{c}\text { Volume ratio } \\
\text { of glycerol }\end{array}$ & $\begin{array}{c}\rho \\
/\left(\mathrm{kg} / \mathrm{m}^{3}\right)\end{array}$ & $\begin{array}{c}\mu \times 10^{3} \\
/\left(\mathrm{m}^{2} / \mathrm{s}\right)\end{array}$ \\
\hline 1 & $0 \%$ & 998 & 1.005 \\
2 & $30 \%$ & 1078 & 3.004 \\
3 & $50 \%$ & 1131 & 8.397 \\
4 & $70 \%$ & 1184 & 35.289
\end{tabular}

In order to study the effects of the operating conditions on the spray cone angles, six injection pressure differentials across the atomizer are selected, i.e., $0.2 \mathrm{MPa}, 0.4 \mathrm{MPa}$, $0.6 \mathrm{MPa}, 0.8 \mathrm{MPa}, 1.0 \mathrm{MPa}$ and $1.2 \mathrm{MPa}$, respectively.
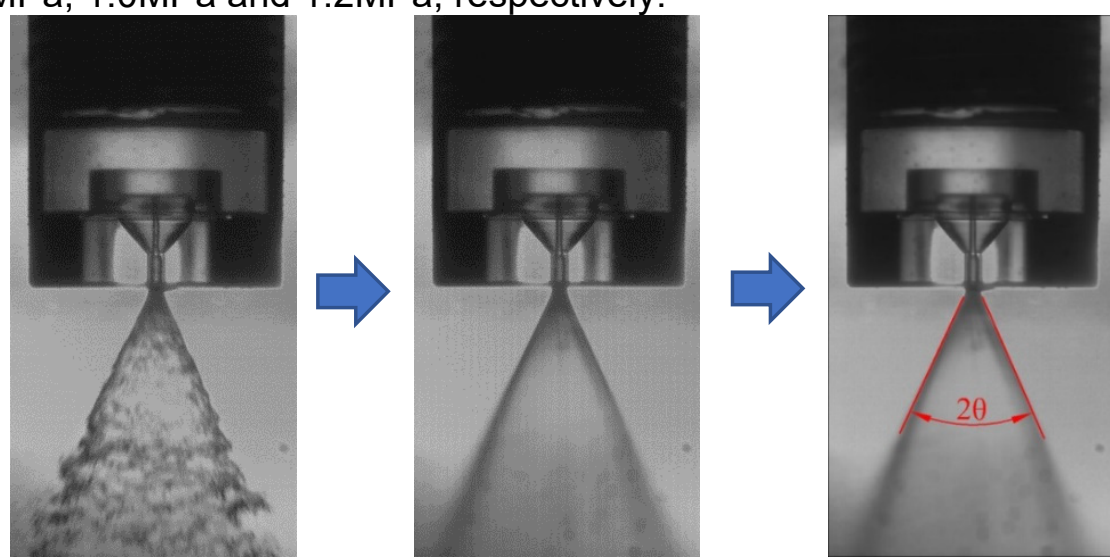

Figure 3. The process to obtain the spray cone angle 
For each test, 500 successive images are obtained. Then these 500 images are averaged to obtain the average spray image. The spray cone angle could be measured from the average spray image. The process is shown in Figure 3 . The uncertainty caused by the measurement is less than $\pm 0.4^{\circ}$.

\section{Results and discussions}

In the experimental tests of the present study, the length of the swirl chamber, liquid viscosity and injection pressure differentials across the atomizer are chosen as the typical parameters of the geometrical parameter, liquid property and operating condition, respectively. A series of these typical parameters are chosen to extend the range of application of the present study.

Table 3 shows the spray cone angles obtained by the experimental tests. The unit of the spray cone angles in Table 4 is deg $\left(^{\circ}\right)$. For the liquid with high viscosity, the standard spray cone could not occur when the length of swirl chamber is long. Thus, some data could not be listed in Table 3.

Table 3 List of the spray cone angles obtained by experimental tests

\begin{tabular}{|c|c|c|c|c|c|c|c|}
\hline \multirow{2}{*}{$\begin{array}{c}\text { Kinetic } \\
\text { viscosity } \\
\times 10^{3} / \\
\mathrm{Pa}^{*} \mathrm{~S}\end{array}$} & \multirow{2}{*}{$\begin{array}{c}\text { Length of } \\
\text { swirl } \\
\text { chamber }\end{array}$} & \multicolumn{6}{|c|}{ Injection pressure differentials across the atomizer / $\mathrm{MPa}$} \\
\hline & & 0.2 & 0.4 & 0.6 & 0.8 & 1.0 & 1.2 \\
\hline \multirow{5}{*}{1.005} & 2.6 & 45.27 & 47.92 & 48.60 & 48.95 & 49.99 & 50.12 \\
\hline & 5 & 39.24 & 42.40 & 43.44 & 44.28 & 44.48 & 44.69 \\
\hline & 10 & 35.89 & 37.35 & 38.91 & 39.65 & 40.68 & 41.39 \\
\hline & 15 & 34.46 & 36.35 & 37.42 & 38.32 & 39.11 & 40.04 \\
\hline & 20 & 31.52 & 33.82 & 35.73 & 36.52 & 37.55 & 38.45 \\
\hline \multirow{5}{*}{3.004} & 2.6 & 41.09 & 43.85 & 44.49 & 45.91 & 46.88 & 47.29 \\
\hline & 5 & 37.60 & 39.69 & 40.98 & 41.73 & 42.48 & 43.10 \\
\hline & 10 & 34.89 & 36.62 & 37.52 & 38.35 & 38.95 & 39.38 \\
\hline & 15 & 30.60 & 32.73 & 34.55 & 36.03 & 36.45 & 37.11 \\
\hline & 20 & 27.93 & 30.87 & 31.40 & 32.85 & 34.27 & 34.85 \\
\hline \multirow{5}{*}{8.397} & 2.6 & 38.19 & 41.67 & 42.96 & 44.30 & 45.29 & 45.82 \\
\hline & 5 & 35.83 & 37.76 & 38.94 & 39.47 & 40.41 & 41.53 \\
\hline & 10 & 31.01 & 33.05 & 34.56 & 36.14 & 36.74 & 37.84 \\
\hline & 15 & & & & 32.90 & 34.03 & 34.50 \\
\hline & 20 & & & & & & 31.37 \\
\hline \multirow{2}{*}{35.289} & 2.6 & 34.60 & 36.56 & 37.27 & 37.95 & 38.49 & 38.85 \\
\hline & 5 & & & & & & 32.33 \\
\hline
\end{tabular}

Based on the data in Table 3, the values of $M, N$ and $Q$ in Eq. (4) could be determined by regression analysis. Finally, the values of 2, $0.1023,5.7419$ are chosen for the values of $M$, $N$ and $Q$. Thus, the expression of Eq. (4) could be expressed as 


$$
2 \theta=\arctan \sqrt{\frac{1+\left(1-61.638 \frac{\rho^{0.1} \mu^{0.2} L_{s}^{0.8}}{\Delta p^{0.1}}\left(1+2 \frac{r_{s}-r_{o}}{L_{s} \tan \alpha}\right)\right)^{2}\left(\left.\frac{W_{\delta_{\theta}}}{U_{\delta_{x}}}\right|_{\text {invscid }}\right)^{2}}{\exp \left\{0.1023\left(\mu \sqrt{\frac{\rho}{\Delta p}}\right)^{0.25} r_{o}^{-0.75} \cdot L_{o}\right\}}}-1
$$

Eq. (5) reveals that the spray cone angles increase with the increasing of the injection pressure differentials across the atomizer quantitatively. Besides, the spray cone angles decrease with the increasing of liquid viscosity and the length of swirl chamber quantitatively. These trends could agree well with the experimental results.

Substituting the values in Tables 1, 2 and 3 into Eq. (5), the spray cone angle predicted by the simplified semi-empirical correlation could be obtained. In the previous studies, Giffen and Massey's correlation and Rizk and Lefebvre's correlation are two typical correlations that are utilized to predict the spray cone angle for viscous liquid. Figure 4 shows the comparison of the spray cone angles predicted by the simplified semi-empirical correlation, Giffen and Massey's correlation [9] and Rizk and Lefebvre's correlation [10] with the experimental results. The expressions of Giffen and Massey's correlation and Rizk and Lefebvre's correlation are written as

$$
\begin{array}{ll}
\tan \theta=0.169 v_{L}^{-0.131} & \text { Giffen and Massey's correlation } \\
2 \theta=6 K^{-0.15}\left(\frac{\Delta P_{L} d_{0}^{2} \rho_{L}}{\mu_{L}^{2}}\right)^{0.11} & \text { Rizk and Lefebvre's correlation }
\end{array}
$$

The $\mathrm{x}$ and $\mathrm{y}$ coordinates denote the spray cone angle measured by experiments and the predicted spray cone angle, respectively. The prediction uncertainties of the simplified semiempirical correlation derived in this paper can be within $\pm 15 \%$ for all cases. The prediction uncertainties of Rizk and Lefebvre's correlation are about $\pm 40 \%$ for all cases. The predicted spray cone angles by Giffen and Massey's correlation remain as constant due to its incapability of accommodating the effects of the variation of geometrical parameters and operating conditions. Thus Giffen and Massey's correlation cannot obtain acceptable prediction results for the present study. Compared with the previous correlations, the one derived from the present study can achieve better prediction accuracy. This simplified semiempirical correlation is available for the quick prediction of the viscous spray cone angle.

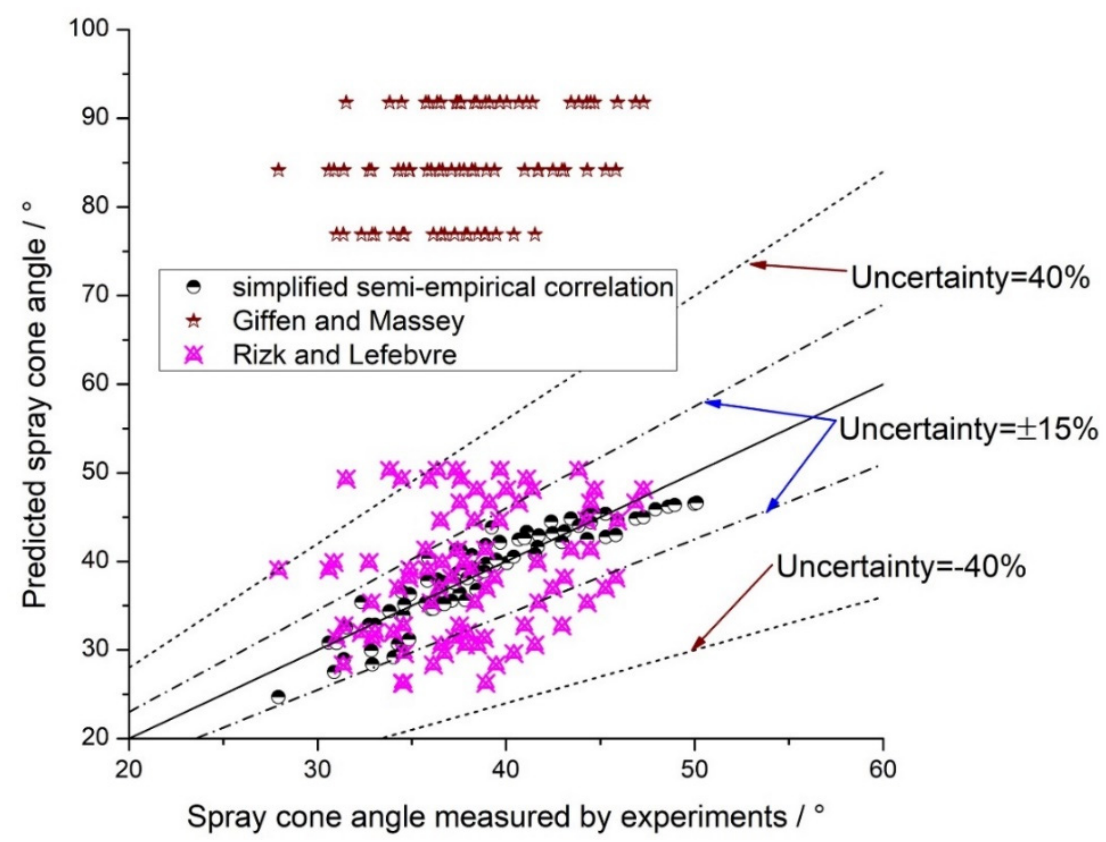


Figure 4. Comparison of the predicted spray cone angles with the experimental results

\section{Conclusions}

In order to achieve quick prediction of the viscous hollow spray cone angle of the pressure swirl atomizers with acceptable prediction accuracy, a simplified semi-empirical correlation is proposed in the present study. Experiments have been conducted to validate this new simplified semi-empirical correlation. In the experimental tests, the length of the swirl chamber, liquid viscosity and injection pressure differential across the atomizer are chosen as the typical parameters of the geometrical parameter, liquid property and operating condition, respectively. A series of these typical parameters are chosen to extend the range of the application of this simplified semi-empirical correlation. Besides, the comparisons of predictions have been done between this new correlation and the previous correlations. Some conclusions could be obtained as follows:

(1) The simplified semi-empirical correlation proposed in the present study could reveal the effects of liquid properties, geometrical parameters and operating conditions on the spray cone angle quantitatively.

(2) The hollow spray cone angles predicted by this simplified semi-empirical correlation agree well with the experimental results. The prediction uncertainties could be within $\pm 15 \%$ for all cases.

(3) Compared with Giffen and Massey's correlation and Rizk and Lefebvre's correlation, this simplified semi-empirical correlation can achieve better prediction accuracy. Thus, this simplified semi-empirical correlation is available for the quick prediction of the viscous hollow spray cone angle.

\section{Acknowledgements}

This work was supported by Chinese National Natural Science Foundation (No. 51906234).

\section{References}

[1] Lefebvre, A. H., 1989, "Atomization and sprays."

[2] Ashgriz, N., 2011, "Handbook of atomization and sprays: theory and applications."

[3] Joshi, JR., 2020, Transactions of the Indian National Academy of Engineering, 5, pp. 269-275.

[4] Fu, W., Liu, Y., Xia, L., Li, M., Song, Z., Hu, H., et al., 2020, EClinicalMedicine, 25, 100478.

[5] Wang, Y., Zhang, Y., Yu, Q., Zhu, K., 2020, Frontiers in Pharmacology, 11, article 966, pp. 1-8.

[6] Mick, P., Murphy, R., 2020, Journal of Otolaryngology - Head and Neck Surgery, 49(29), pp. 1-10.

[7] Chinn, J. J., 2009, Atomization and Sprays, 19(3), pp. 263-282.

[8] Sun, L., Huang, Y., Liu, Z., 2017, Atomization and Sprays, 27(9), pp. 771-790.

[9] Giffen, E., Massey, B. S., 1950, Report 1950/5, Motor Industry Research Association, United Kingdom.

[10] Rizk, N. K., Lefebvre, A. H., 1987, International Journal of Turbo and Jet Engines, 4(1-2), pp. 65-74.

[11] Zhang, T., Dong, B., Chen, X., Qiu, Z., Jiang, R., Li, W., 2017, Applied Thermal Engineering, 121, pp. 984-991.

[12] Buckingham, E., 1915, Nature, 96, pp. 396-397.

[13] Rivas, J. R. R., Pimenta, A. P., Rivas, G. A. R., 2014, Atomization and Sprays, 24(2), pp. 97-114.

[14] Liu, Z., Huang, Y., Sun, L., 2017, International Journal of Hydrogen Energy, 42(29), pp. 18649-18657. 